\title{
Angler peptides: macrocyclic conjugates inhibit p53:MDM2/X interactions and activate apoptosis in cancer cells
}

Grégoire J-B. Philippe, ${ }^{1,2}$ Anna Mittermeier, ${ }^{3}$ Nicole Lawrence, ${ }^{1,2}$ Yen-Hua Huang, ${ }^{1,2}$ Nicholas

D. Condon, ${ }^{1}$ Alexander Loewer, ${ }^{3}$ David J. Craik, ${ }^{1,2}$ Sónia T. Henriques. ${ }^{1,2,4 *}$

${ }^{1}$ Institute for Molecular Bioscience, The University of Queensland, Brisbane, Queensland, 4072, Australia

${ }^{2}$ Australian Research Council Centre of Excellence for Innovations in Peptide and Protein Science, The University of Queensland, Brisbane, Queensland, 4072, Australia

${ }^{3}$ Technische Universität Darmstadt, 64287, Darmstadt, Germany

${ }^{4}$ Queensland University of Technology, School of Biomedical Sciences, Institute of Health \& Biomedical Innovation and Translational Research Institute, Brisbane, Queensland, 4102, Australia

*To whom correspondence should be addressed:

Sónia T. Henriques, E-mail: sonia.henriques@qut.edu.au 


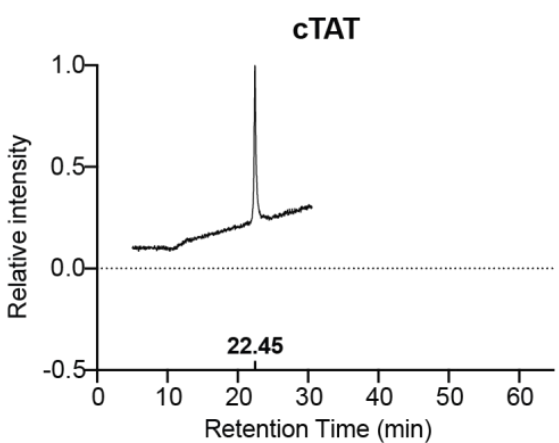

cTAT-KD3

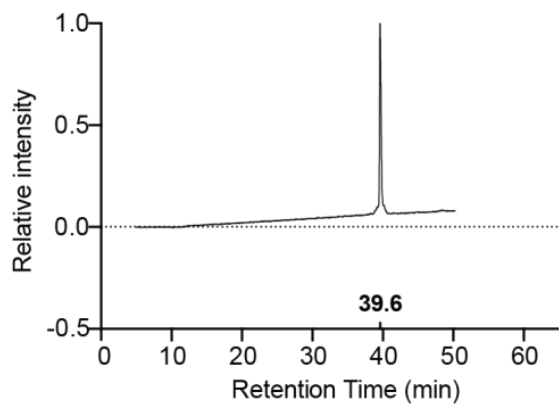

CCPP12-KD3

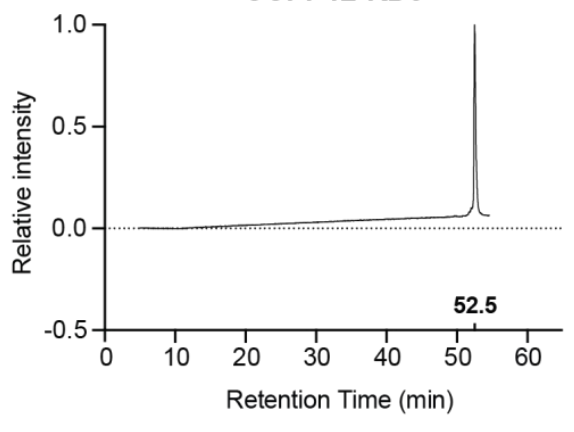

[F3A]KD3*

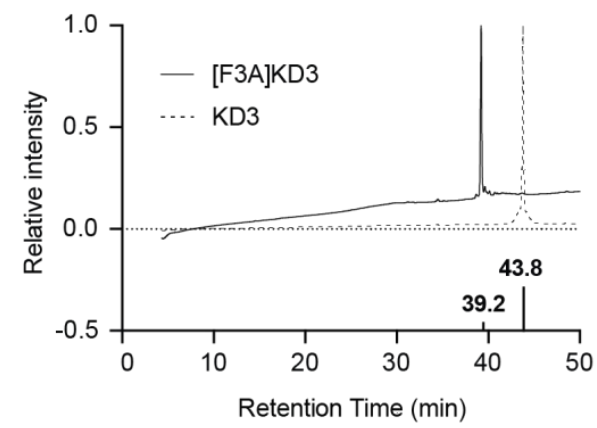

cR10

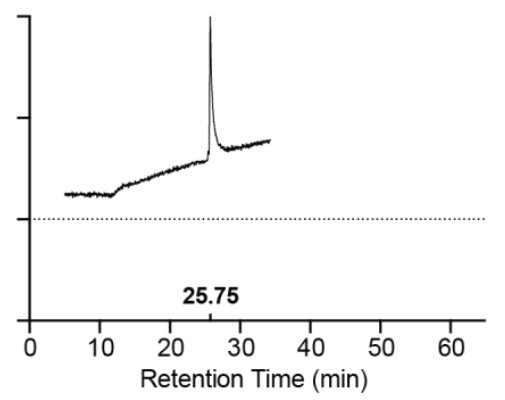

cR10-KD3

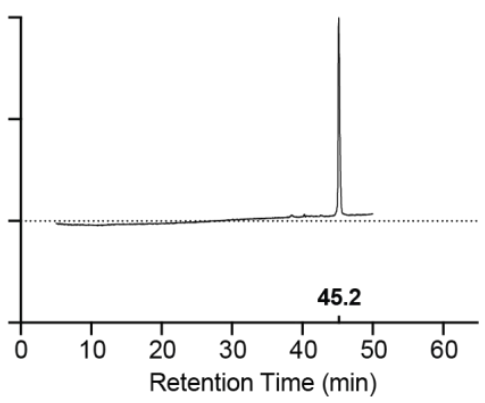

ATSP6935

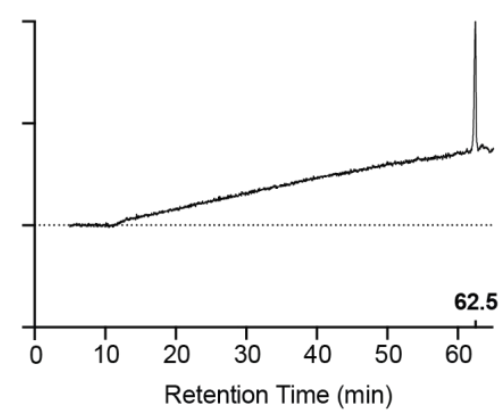

KD3

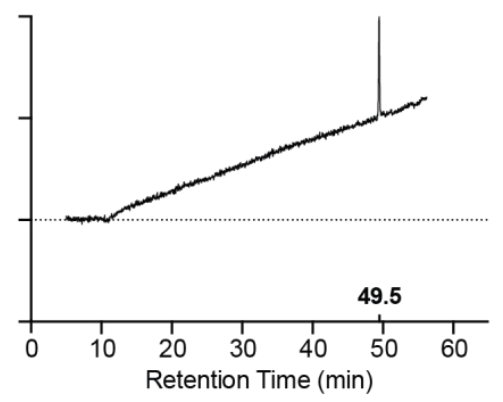

p18-KD3

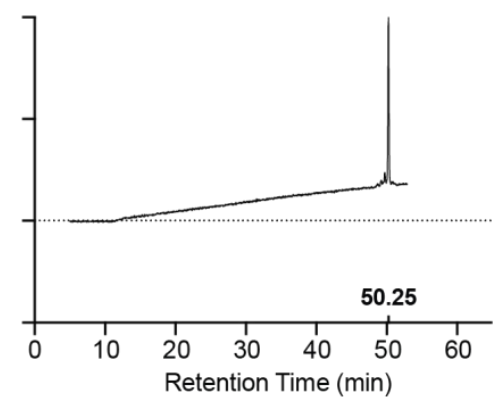

cPF4PD-KD3

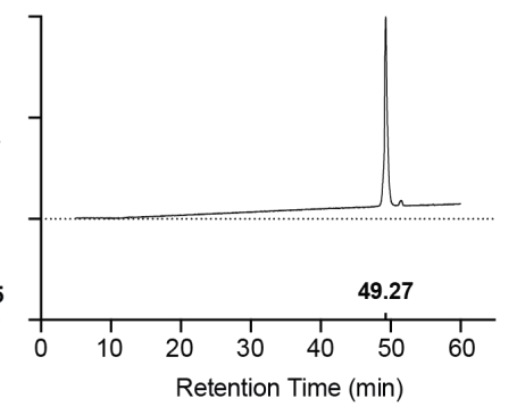

cR10-[F3A]KD3*

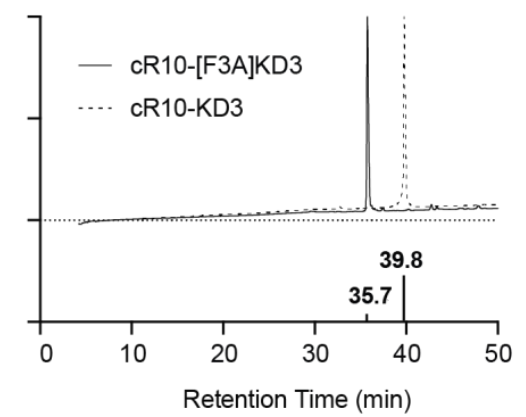

Figure S1. Analytical RP-HPLC chromatograms obtained with peptides used in this study and detected by absorbance at 215 $\mathrm{nm}$. Peptides were eluted from the column using a $1 \%$ gradient of solvent B $(90 \%(\mathrm{v} / \mathrm{v})$ acetonitrile $+0.05 \%$ trifluoroacetic acid) in solvent $\mathrm{A}(0.05 \%(\mathrm{v} / \mathrm{v})$ trifluoroacetic acid). Absorbance was normalised (relative intensity) to the peak, which corresponds to the retention time of the peptide (indicated in bold). The first 5 minutes following injection on the column were not included. *[F3A]KD3 and cR10-[F3A]KD3 chromatograph were acquired on a different HPLC and column, and were compared directly to their corresponding parent peptide on that system. 


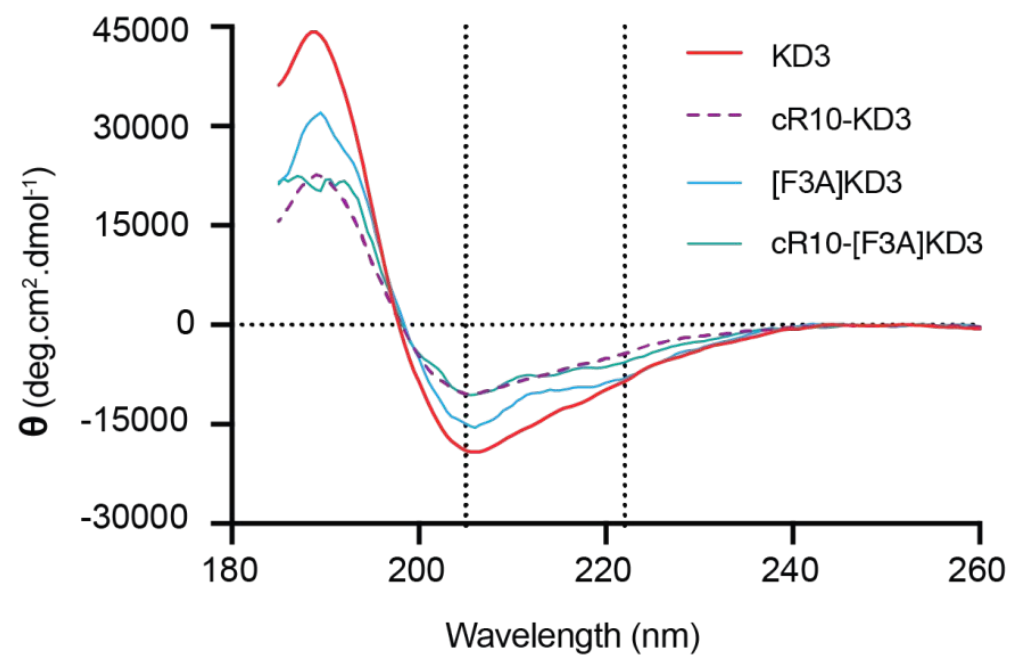

Figure S2. Helicity of KD3 and mutant [F3A]KD3 and the corresponding cR10-angler peptides. The secondary structure was assessed using CD spectroscopy and the percentage of helical conformation (KD3 30\%, cR10-KD3 15\%, [F3A]KD3 29\%, and cR10[F3A]KD3 19\%) was estimated as described in Figure 2. 
A

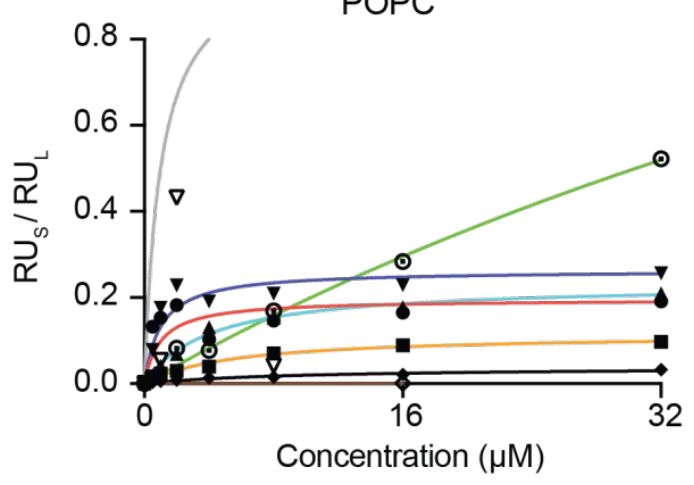

POPC-POPS

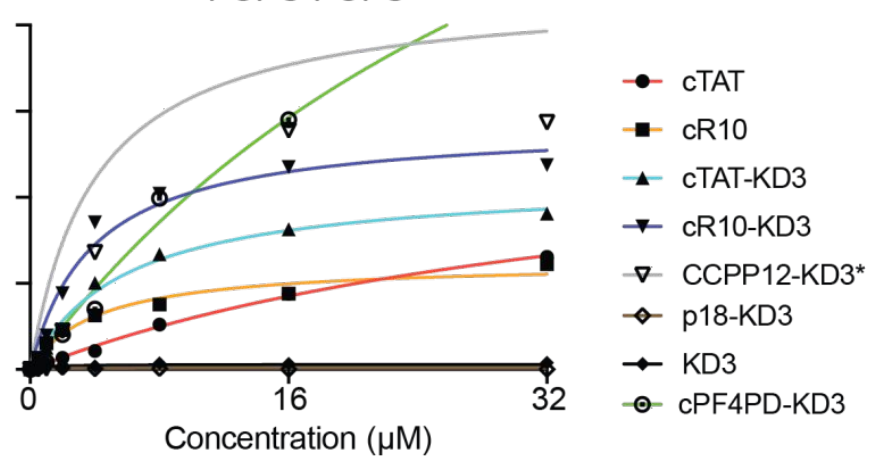

B

\begin{tabular}{lccccc} 
B & \multicolumn{2}{c}{$\mathrm{POPC}$} & & \multicolumn{2}{c}{$\mathrm{POPC} / \mathrm{POPS}(4: 1)$} \\
\cline { 2 - 3 } Peptide & $\mathrm{K}_{\mathrm{p}} \times 10^{3}$ & $\mathrm{P} / \mathrm{L}_{\max }$ & & $\mathrm{K}_{\mathrm{p}} \times 10^{3}$ & $\mathrm{P} / \mathrm{L}_{\max }$ \\
\hline KD3 & $2.2 \pm 0.5$ & 0.017 & - & & - \\
CTAT & $112.5 \pm 76.4$ & - & & $8.7 \pm 0.5$ & 0.26 \\
CR10 & $11.9 \pm 1.8$ & 0.051 & & $35.5 \pm 6.4$ & 0.11 \\
CTAT-KD3 & $32.8 \pm 3.3$ & 0.102 & & $43.8 \pm 0.2$ & 0.194 \\
CR10-KD3 & $151.2 \pm 50.5$ & - & & $85.4 \pm 17.4$ & 0.252 \\
CPF4PD-KD3 & $12.3 \pm 1.8$ & - & & $29.9 \pm 3.2$ & 0.88 \\
CCPP12-KD3 & - & - & & $128.0 \pm 146$ & 0.39 \\
p18-KD3 & 0.0 & - & & 0.0 & -
\end{tabular}

*The peptide disrupted the lipid bilayer.

Figure S3. Membrane partition constant $\left(\mathrm{K}_{\mathrm{p}}\right)$ of peptides determined by SPR. The partition constant is the ratio of molecules distributed between the aqueous and lipid phases and relates to the affinity of the peptide for lipid bilayer composed of POPC, or POPC/POPS (4:1). (A) The fitted dose responses curves using response unit of the solute $\left(\mathrm{RU}_{\mathrm{S}}\right)$ and the response unit of the lipid $\left(R U_{L}\right)$ were used to calculate $K_{p}$ as before. ${ }^{1}$ (B) $K_{p}$ and the maximum ratio between peptide/lipid $\left(\mathrm{P} / \mathrm{L}_{\max }\right)$ were determined as before. ${ }^{1-2}$ Value not displayed could not be calculated using the partition constant equation. 


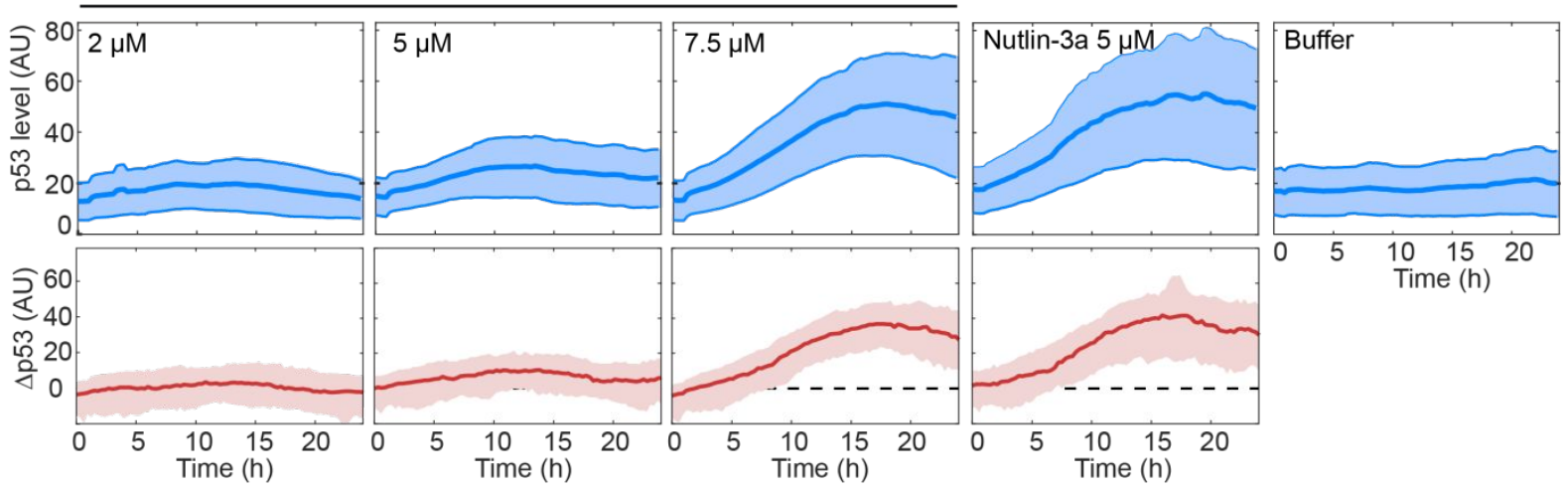

B

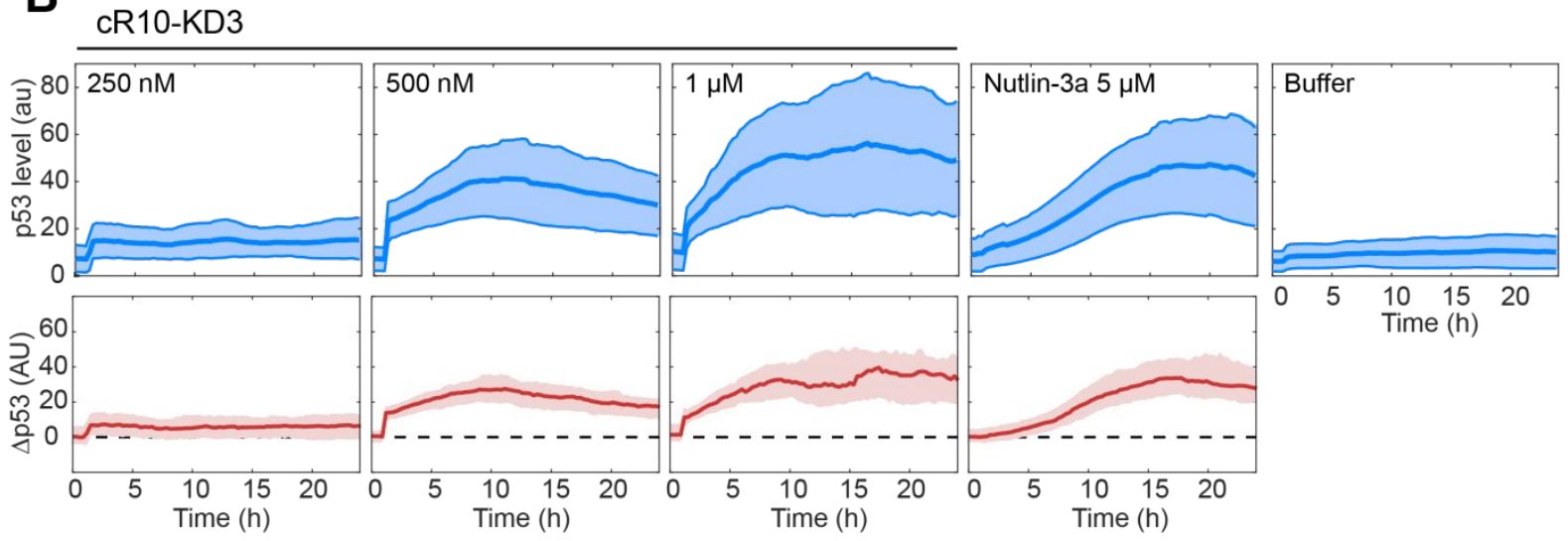

Figure S4. Activation of the p53 pathway in HCT116 reporter cells. The average nuclear fluorescence intensities in HCT116 reporter cells expressing p53-mVenus. Fluorescence emission intensities directly correlate with p53 levels. The indicated peptides were added after $1 \mathrm{~h}(\mathrm{t}=1)$ and fluorescence emission intensity (excitation at 500/24 nm and emission at 542/27 nm) was monitored for $24 \mathrm{~h}$ in total. The mean fluorescence emission intensity (blue bold line) as well as $25^{\text {th }}$ to $75^{\text {th }}$ percentiles (blue shaded area) are shown for each condition ( $\mathrm{n}>38$ cells). The effect size (difference in mean levels between treated cells and control) is represented by the red curve and the $95 \%$ confidence interval based on permutation testing is indicated by the red shaded area. Increase in p53 protein levels show the binding of the tested peptide to MDM2 and MDMX within cells and the activation of the p53 pathway. Nutlin-3a is a small molecule that efficiently binds to MDM2. cR10-KD3 (B) triggers more activation of p53 pathway at $500 \mathrm{nM}$ in HTC166 cells, than in MCF7 cells (see Figure 5 in text). On the other hand, cTATKD3 (A) only activated p53 pathway in HTC116 cells at $7.5 \mu \mathrm{M}$, which was much higher than the concentration needed in MCF7 cells ( $1 \mu \mathrm{M}$, see Figure 5 in main text). Data from (A) and (B) were acquired in two separate experiments, each with the same subset of controls for comparison. 
A
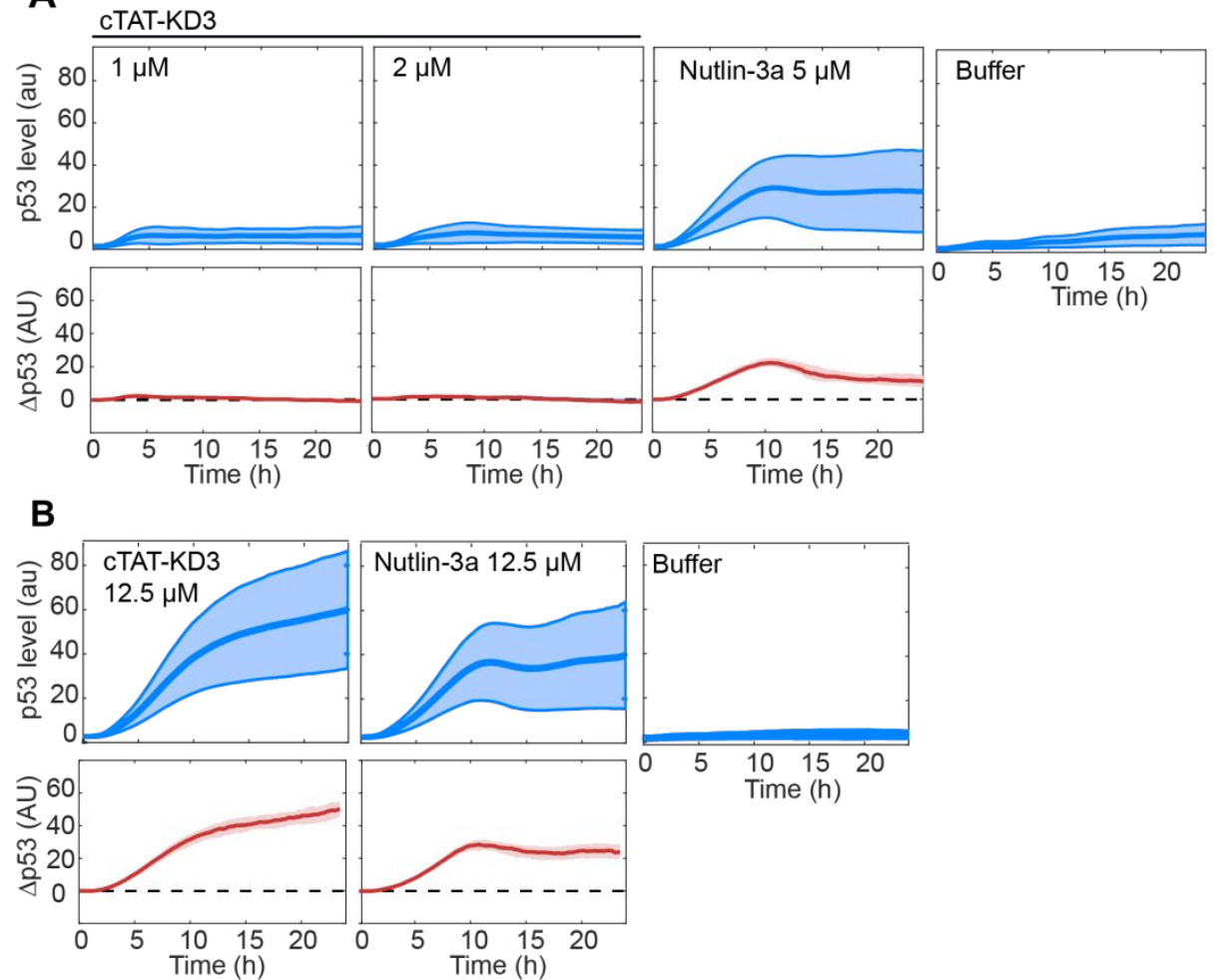

ime $(\mathrm{h})$

C

CR10-KD3

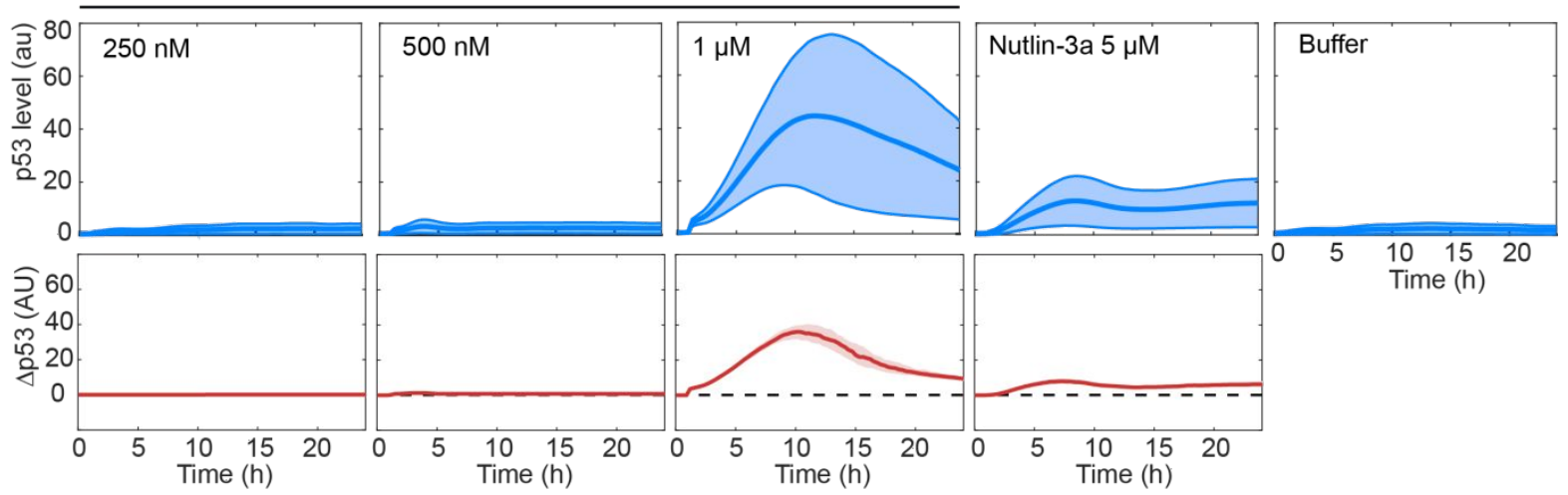

Figure S5: p53 activation in a A549 reporter cell line. Time-lapse microscopy was used to measure the average nuclear fluorescence intensities in A549 cells expressing p53-mVenus. Fluorescence emission intensities directly correlate with p53 levels. The indicated peptides were added after $1 \mathrm{~h}(\mathrm{t}=1)$ and fluorescence emission intensity (excitation at 500/24 nm and emission at $542 / 27 \mathrm{~nm}$ ) was monitored for $24 \mathrm{~h}$ in total. The mean fluorescence emission intensity (blue bold line) as well as $25^{\text {th }}$ to $75^{\text {th }}$ percentiles (blue shaded area) are shown for each condition ( $\mathrm{n}>546$ cells). The effect size (difference in mean levels between treated cells and control) is represented by the red curve and the $95 \%$ confidence interval based on permutation testing is indicated by the red shaded area. Accumulation of $\mathrm{p} 53$ protein demonstrates the binding of the tested peptide to MDM2 and MDMX within cells and the activation of the p53 pathway. Nutlin-3a is a small molecule that efficiently binds to MDM2. cTAT-KD3 (A,B) was only active at $12.5 \mu \mathrm{M}$ in A549 cells which is a higher concentration than that required to activate p53 in the other cell lines. cR10-KD3 (C) did not activate the p53 pathway at $500 \mathrm{nM}$ but strongly activated it $1 \mu \mathrm{M}$. Data were acquired in three separate experiments (A,B,C), each with their subset of controls. Together Figures 4, S4 and S5 suggest that cR10-KD3 is able to activate the p53 pathway consistently at $1 \mu \mathrm{M}$, whereas significant differences appear between cell lines with cTAT-KD3 (activation at $1 \mu \mathrm{M}$ in MCF7, $7.5 \mu \mathrm{M}$ in HCT116 and $12.5 \mu \mathrm{M}$ in A549). 

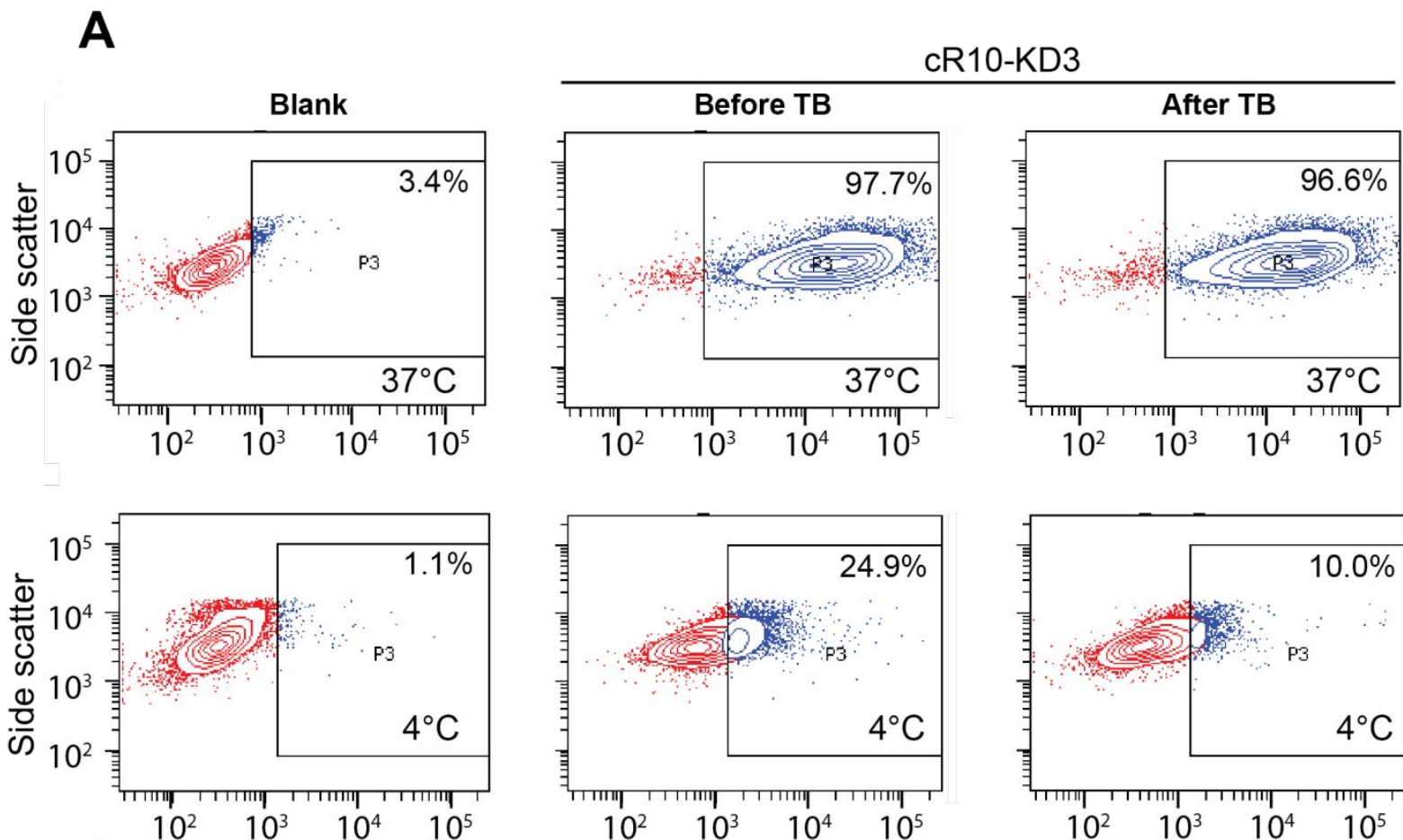

Fluorescence intensity

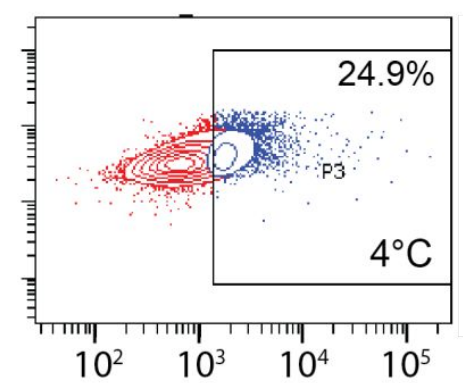

Fluorescence intensity

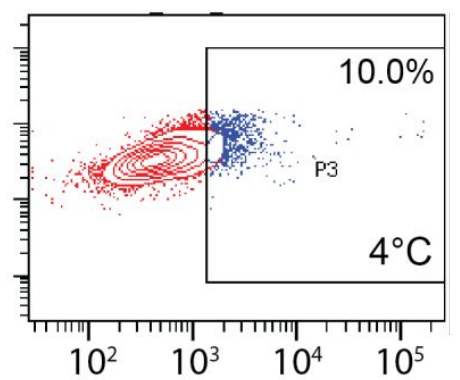

Fluorescence intensity
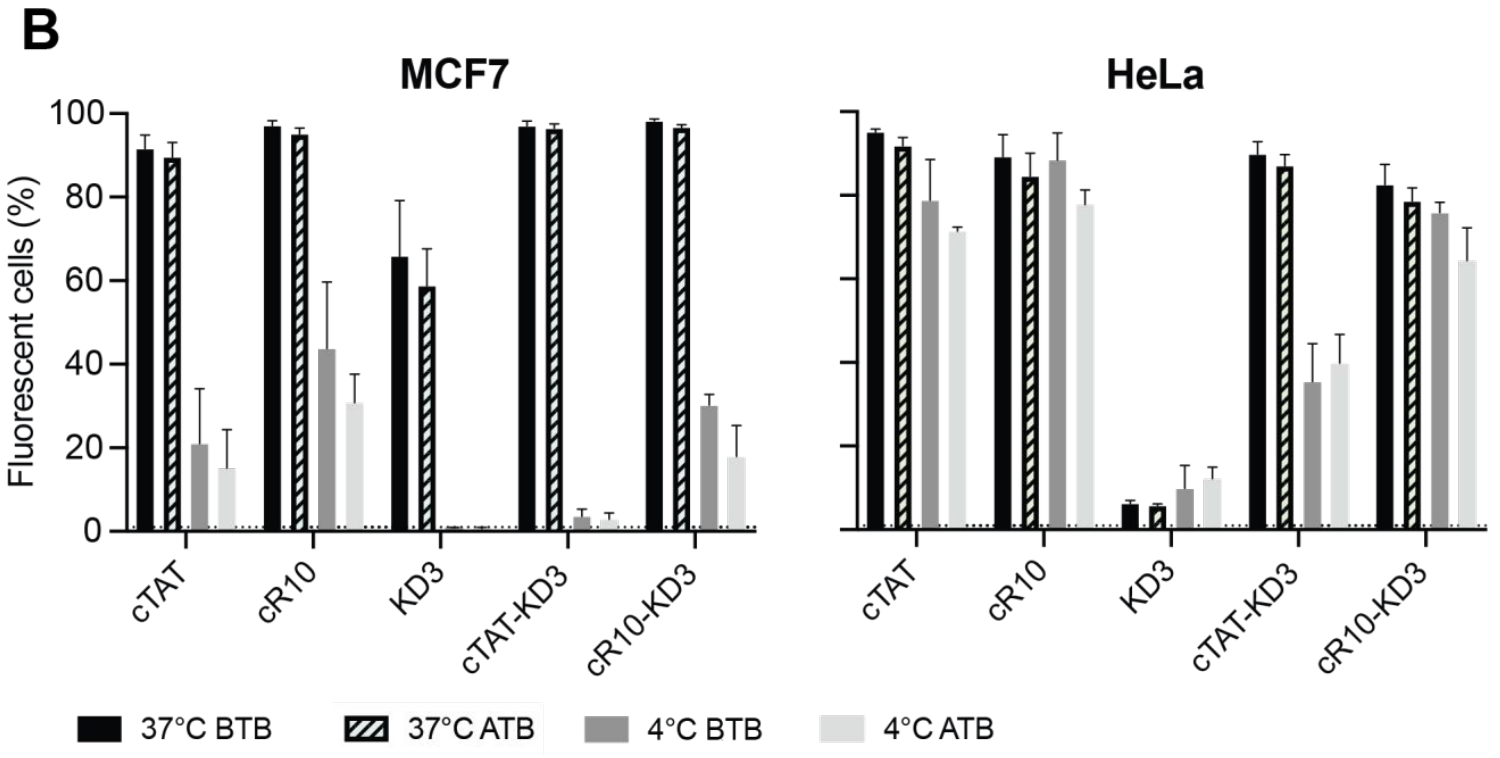

Figure S6: Percentage of cells with internalized labelled-peptide. (A) A dotplot of the fluorescence intensity in function of the side scatter light is used to define cells with internalized labelled-peptides and each dot represents one cell. Dots that fall in the gate (P3) are cells that are fluorescent. The internalisation of Alexa Fluor 488-cR10-KD3 in MCF7 cells was chosen as an example of internalisation at both $37^{\circ} \mathrm{C}$ and $4{ }^{\circ} \mathrm{C}$. After treatment with Trypan blue (TB), the fluorescence of membrane bound peptide and lysed cells is quenched. (B) The graph indicates the percentage of fluorescent cells, as quantified using dotplots identified in (A) for each peptide in the four tested conditions and in the two tested cell lines. BTB stands for before TB, ATB stands for after TB. 


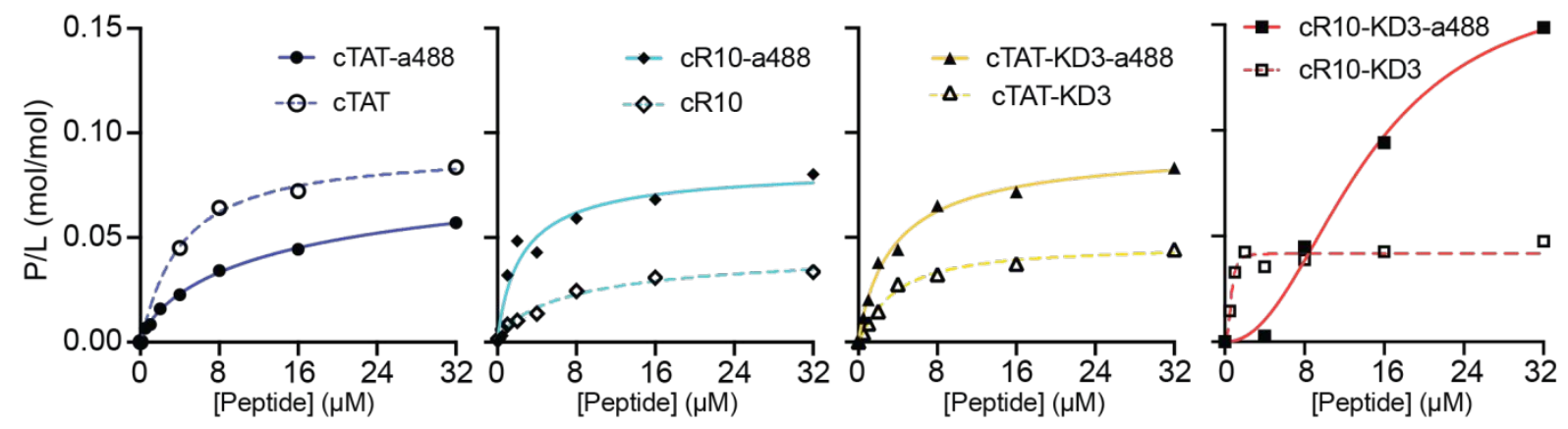

Figure S7. Binding of unlabeled and Alexa Fluor 488®(A488) labeled peptides to lipid bilayers composed of POPC monitored using SPR. A488 labeled peptides (dashed lines) cTAT-a488, cR10-a488 and cTAT-KD3-a488 have different membranebinding affinity, when compared to unlabelled peptides (solid lines), but retain weak affinity for POPC bilayers, especially at the lower $\mu \mathrm{M}$ concentrations $(<4 \mu \mathrm{M})$ that are used in biological assays in this study. The A488 labelled cR10-KD3 has increased membrane-binding affinity at $>8 \mu \mathrm{M}$.

\section{REFERENCES SI}

1. Figueira, T. N.; Freire, J. M.; Cunha-Santos, C.; Heras, M.; Goncalves, J.; Moscona, A.; Porotto, M.; Salome Veiga, A.; Castanho, M. A., Quantitative analysis of molecular partition towards lipid membranes using surface plasmon resonance. Sci. Rep., 2017, 7, 45647.

2. $\quad$ Philippe, G. J.; Gaspar, D.; Sheng, C.; Huang, Y. H.; Benfield, A. H.; Condon, N. D.; Weidmann, J.; Lawrence, N.; Lower, A.; Castanho, M.; Craik, D. J.; Troeira Henriques, S., Cell Membrane Composition Drives Selectivity and Toxicity of Designed Cyclic Helix-Loop-Helix Peptides with Cell Penetrating and Tumor Suppressor Properties. ACS Chem. Biol., 2019, 14, 2071-2087. 